$1-1995$

\title{
Basics of research (Part I): Why conduct clinical research and how to get started?
}

\author{
Edward A. Panacek \\ University of California, Davis \\ Cheryl Thompson \\ University of Nebraska Medical Center, cbthompson@unmc.edu
}

Tell us how you used this information in this short survey.

Follow this and additional works at: https://digitalcommons.unmc.edu/con_articles

Part of the Nursing Commons

\section{Recommended Citation}

Panacek, Edward A. and Thompson, Cheryl, "Basics of research (Part I): Why conduct clinical research and how to get started?" (1995). Journal Articles: College of Nursing. 14.

https://digitalcommons.unmc.edu/con_articles/14

This Article is brought to you for free and open access by the College of Nursing at DigitalCommons@UNMC. It has been accepted for inclusion in Journal Articles: College of Nursing by an authorized administrator of DigitalCommons@UNMC.For more information, please contact digitalcommons@unmc.edu. 


\title{
Basics of Research (Part I): Why Conduct Clinical Research and How to Get Started?
}

\author{
Edward A. Panacek, MD, ${ }^{1}$ Cheryl Thompson, RN, MSN, PhD²
}

\author{
1. Division of Emergency Medicine and \\ Clinical Toxicology, University of \\ California, Davis, California \\ 2. University of Utah, College of Nursing, \\ Salt Lake City, Utah
}

Key Words: clinical investigation, clinical research, research

Address for correspondence: Edward A. Panacek, MD, U.C. Davis Medical Center, Division of Emergency Medicine, 2315

Stockton Blvd., Sacramento, CA 95817
Though we often do not consciously acknowledge it, most of our clinical decisions have their basis in previous published research studies. Not all of that clinical research was conducted with rigorous scicntific mcthodology and sometimes conclusions were drawn that are unsupported by actual data. Nonetheless, all clinical studies are potentially very powerful for shaping the way in which medical care is delivered. Even a single article can radically change clinical practices or understanding of a disease process. It is sometimes astounding, and embarrassing, to see how dogma that had been accepted by generations of clinicians is suddenly challenged and disproved by an investigator who simply seeks to "know the truth." For example, two decades of ACLS courses taught the empirical use of sodium bicarbonate in the resuscitation of all victims of cardiac arrest. It was not until studies showed that bicarbonate may actually cause harm by worsening cellular acidosis ${ }^{1}$ that the 1992 guidelines dropped it from routine use. ${ }^{2}$ In the air medical field, it was "common knowledge" that electrical defibrillation/ cardioversion was not safe in an airborne helicopter until a study published in 1989 proved that to be nonsense. ${ }^{3}$

Publishing in professional literature and adding to the body of medical knowledge is a rewarding contribution to make to one's profession. However, conducting credible research is not an easy task; it's plain hard work. Unfortunately, too often investigators are willing to accept projects that are seriously flawed, rather than commit the time and resources to do them properly. Once such studies are published, their conclusions are often quoted without a close examination of the methodology. Refuting the conclusions of faulty studies takes a great deal of time and effort. Too often the conclusions go unchallenged and inappropriate clinical practices can result. Most flawed studies in the medical literature are not the result of dishonesty or laziness but due to investigator ignorance. Unless individuals pursue a $\mathrm{PhD}$, or a researchoriented fellowship or similar training program, they may never receive any formal education in research methodology. As a result, most people involved with clinical research are relatively selftaught. In the course of "learning by doing," some never learn the basics, others develop bad habits and almost all can benefit from a more structured, formal education in proper research design and methodology.

We will address this need with this multipart series in clinical research. Over the next several issues, we will provide a series of articles on the basics of performing research that fill in some of those educational holes, while also serving as a "stand-alone" set of articles for the novice who wishes to become involved in clinical research. This series of articles covers the entire research process-formulating a research question, selecting a research design, fleshing out a protocol, understanding the basics of statistics, and, ultimately, presenting or publishing the results. It is our hope that 
this series of articles will bring a better appreciation for the difficulty of doing research properly, while also avoiding some of the pitfalls encountered when first involved in investigative studies. When it comes to research, everyone starts as a beginner, and the challenges can seem overwhelming. However, it is important to emphasize that the rewards of performing good research clearly outweigh the frustrations and difficulties.

There is certainly more than one way to approach a research project. This becomes painfully obvious when reading multiple articles or textbooks on the subject. Each textbook contains slightly different recommendations and often uses different systems for categorizing research designs, and so on. The important point is not to focus on a single classification system or approach, but to understand some of the basic underlying principles and to learn to use the tools that are most relevant to your setting, background and experience. Regardless of the system used, a systematic approach to performing research is important, and there are some fundamental "rules" that have stood the test of time. This series of articles presents a relatively common accepted framework for approaching research, and emphasizes a practical approach.

\section{How to Get Started}

One of the great ironies of research is that when you first get started in clinical practice you have the grcatest amount of extra time, but the least number of research ideas. As you become more experienced, you find that there are dozens or literally hundreds of research ideas, but not nearly enough time to pursue even the most important ones. Properly performed research is a lot more work than most people anticipate, and, at some point, almost every research project has an element of drudgery. As such, it is important to pick an area of true interest. If researchers have a passion to know more about an area or the answer to a specific question, that enthusiasm will carry them through the most difficult stages of data collection and analysis. If you are unsure where to start, there are a number of excellent sources of research questions all around us:
1. Your own clinical practice is often the best source. Every day you encounter gaps in your own medical knowledge. For example:

a. Clinical observations you have made that do not fit "the textbook."

b. Frustrations you feel when confronted with a patient condition for which available treatment is very incomplete.

c. Treatment protocols that "everyone uses" but for which no one can demonstrate a scientific basis.

2. Discussion with professional colleagues.

Often discussions of an individual patient or one's clinical practices will identify areas appropriate for clinical investigation.

3. Inspiration from other investigators at professional meetings.

Often research presentations include a discussion of "future directions for research" or "questions not yet answered." Even when the investigator claims already to be working on these questions, the reality is they most often are not. Feel free to seek out these individuals and further discuss areas of mutual interest. Most researchers love discussing their studies and quickly generate several ideas worthy of further investigation.

4. Identification of gaps in the medical literature.

While doing a literature review on a given subject, it quickly becomes apparcnt that there are aspects of the topic that are well known and other areas in which knowledge is severely lacking. Often, recommended clinical practices are supported by anecdote, not by actual science. In addition, most research articles spend time discussing "the limitations of this study" or identifying appropriate related areas for further investigation. These are usually discussed in the last two or three paragraphs of the article.

After some practice, research questions can be identified practically every time you see a patient. All that is required is an inquisitive mind, a willingness to question dogma and an appropriate base of experience from which to make observations.

\section{Refining the Research Question}

Once you have initially identified an area of research interest, or a preliminary research question, the experience of a "stream of ideas" is very common. Instead of just one research question, literally dozens of related questions may come to mind. Should you look at the research question this way, or that way, in this patient population, or in that patient population? Is it more important to look at antecedent history and potential etiology, or natural course and outcome? Several related or "tag-a-long" type research questions may occur to you. This process is very important in helping to sort through options, helping to better understand the source of interest and further defining the exact nature of the research question. However, at this stage there is a great temptation to broaden the research question and this temptation should be strongly resisted. It is important to sort through these various ideas and then focus your research question down to a specific area. Too many researchers start out with an ill-defined project with no testable hypotheses. If this is not rectified in the early stages, the entire study will be nebulous and waste precious investigator time and resources.

Another potential obstacle, at this point, is the inability to proceed from a general area of interest/concern and convert it into a specific research question. Before any further planning efforts can bcgin, research must have a primary focus. One way to achieve this is to break the topic area into constituent parts. Separately write down each aspect of the topic and transform each of those into individual questions. Rank the questions in order of importance or greatest interest. This way you can single out one or two to build into your proposed project. Each study should have only one primary question. It is common to also have one, two or, occasionally, three secondary questions. However, these are meant to be complementary and should not be allowed to detract from the primary research question itself.

After the initial "brainstorming" about your research question, it is time to become more structured. At this point, prepare to write down the exact pro- 
posed research question in a single, understandable sentence. The question can be written in a number of different ways and it need not yet fit the form of a "null hypothesis." It should be simplea single sentence-and written in the form of a question. The vast majority of clinical research questions will fit one of the following categories:

1. An evaluation of the accuracy or usefulness of a diagnostic test.

2. An evaluation of the effectiveness of a new or competing therapy or device.

3. An evaluation of the etiology of a clinical condition.

4. A description of the natural course or outcome of a medical condition.

5. $\Lambda \mathrm{n}$ analysis of clinical decision making or cost effectiveness.

6. Description of current practice, emerging trends or a new observation which is not previously described.

Except for this last area, which is purely observational or descriptive, all of the other categories of research questions benefit from an appropriate research design and scientific approach. Once you have a draft research question, it is easy to make the mistake of expediency rather than selecting a more scientific approach to the project. Too often investigators settle for what is measurable or readily available, rather than what is important. Now you should have a good sense of what type of research question you wish to ask. To further refine the question, and help determine whether it is a practical project to undertake, there are several aspects to consider. The characteristics of a good research question have been well described by Doctors Stephen Hulley and Steven Cummings in their book, "Designing Clinical Research," where they use the acronym of a FINER research question:

\section{Feasible}

Does your practice have an adequate number of potential research subjects and are there the appropriate resources available to perform the project? Are the end points measurable? Is the study manageable in scope, financial costs and in terms of your own time?

\section{Interesting}

Is this a topic that truly interests you and for which you have a "passion to know?"

\section{Novel}

Is this a new idea or would it simply be "reinventing" a well established wheel? Does it add to or refute previous findings or provide new observations? Does it help confirm previous studies which remain controversial?

\section{Ethical}

Would performing this project represent ethical clinical and investigative practices?

\section{Relevant}

Would the results of this project be relevant in terms of impacting current medical care or the direction of future research? If, regardless of the results of your study, the response of most of your colleagues would be "So what?" then it is probably not a project worthy of your time and efforts.

The research question is the objective of the study-the gap in medical knowledge that you hope to resolve. All of the subsequent research efforts will be guided by the focus of the research question; therefore, be careful in formulating that question. In many ways, this question becomes the compass by which all subsequent decisions are made. Too often novice investigators do not appreciate fully this fact and rush through the process of outlining their research question. Particularly at this stage of a research project, being on the right track is more important than speed. As Sir Francis Bacon pointed out so eloquently, "The lame man who keeps to the right road outstrips the runner who takes a wrong one. Nay, it is obvious that when a man runs the wrong way, the more active and swift he is the further he will go astray."

At the very least, discuss your research question with an experienced and respected colleague. Far better, if the opportunity exists, present your proposed question before an audience of critical peers. Some institutions have a "research in progress" conference that serves this purpose very well. For example, at the U.C. Davis Emergency Mcdicinc Residency, completing a re- search project is a requirement of the program, but the residents are usually inexperienced in this area. A year before the projects are due, each resident must make a public presentation that covers their intended research question and interpretation of the relevant literature. The audience, consisting of other residents and the faculty, is encouraged to constructively criticize each presentation. This process helps to refine the projects while in the planning stages. If such opportunities are unavailable, informally discussing the question at an appropriate educational or administrative meeting can be very helpful.

\section{Taking the Next Step}

Now you have a research question that is highly specific, though not necessarily finalized. You have started the planning process that is the most important part of any research study. In research endeavors, $90 \%$ of your time should be spent on planning and $10 \%$ on actually performing the study. The next step is to review systematically, or re-review, the literature relevant to your focused research question. As a result of that literature review, finalize your research question in the form of a hypothesis. That hypothesis will consist of two variables and express a proposed relationship between those variables.

The following is an example of taking a general interest area and refining it into potentially good and poor research questions, and finally into a focused question.

Area of research interest:

- Flight crew experiences with endotracheal intubation.

Examples of poor or nebulous questions:

- Do flight nurses do a good job with in-field intubations?

- What is the nature of flight nurse intubation experience?

Examples of better worded questions:

- Does the intubation success rate of flight nurses equal that of flight physicians?

- Do flight nurses have the same intubation success rate in the field that they do in the emergency department or the operating room? 
Examples of highly specific research questions:

- Does the use of neuromuscular blocking agents increase the success rate of in-field intubations by flight nurses?

- Is the intubation success rate better when performed in the helicopter than when performed outside the craft, at the scene?
Specific research questions can be converted more easily into an actual research hypothesis, which can then be tested using a study design and protocol.

Congratulations on taking the first and most important step to being a researcher. Though there are many roadblocks ahead, there is tremendous personal satisfaction in performing credible research. This series will try to help with each of the steps and point out some of the pitfalls. In addition, if you want to read about this subject in greater depth, there are a number of excellent textbooks available. Listed below are some of the best for the beginning researcher.

\section{References}

1. Grundler W, Weil MH, Yamaguchi M, Michaels $\mathrm{S}$ : The paradox of venous acidosis and arterial alkalosis during CPR. CHEST 1984;86:282.

2. Emergency Cardiac Care Committee, American
Heart Association: Guidelines for cardiopulmonary resuscitation and emergency cardiac care. JAMA 1992; 268:2171-2302.

3. Dedrick DK, Darga A, Handis D, et al: Defibrit lation safety in emergency helicopter transport. Ann Emerg Med 1989;125-127.

\section{Recommended Texts}

1. Bailey DM. Research for the Health Professional: A Practical Guide. 1991. FA. Davis, Co., Philadelphia (Great for the novice. Lots of examples and sample worksheets.)

2. Hulley SB, Cummings SR. Designing Clinical
Research. Williams and Wilkins. Baltimore, MD. 1988. (Rather comprehensive and sophisticated, but still user friendly. Has a public health, epidemiologic orientation.)

3. Okolo EN. Health Research Design and Method- ology. 1990. CRC Press, Inc., Boca Raton, FL. (Focus on writing proposals, interpreting data and reporting findings. Many practical examples and not as much theory.)

Editor's Note: The next installment in this series will be "Reviewing the Literature" and will appear in the April-June issue of the Air Medical Journal. 